
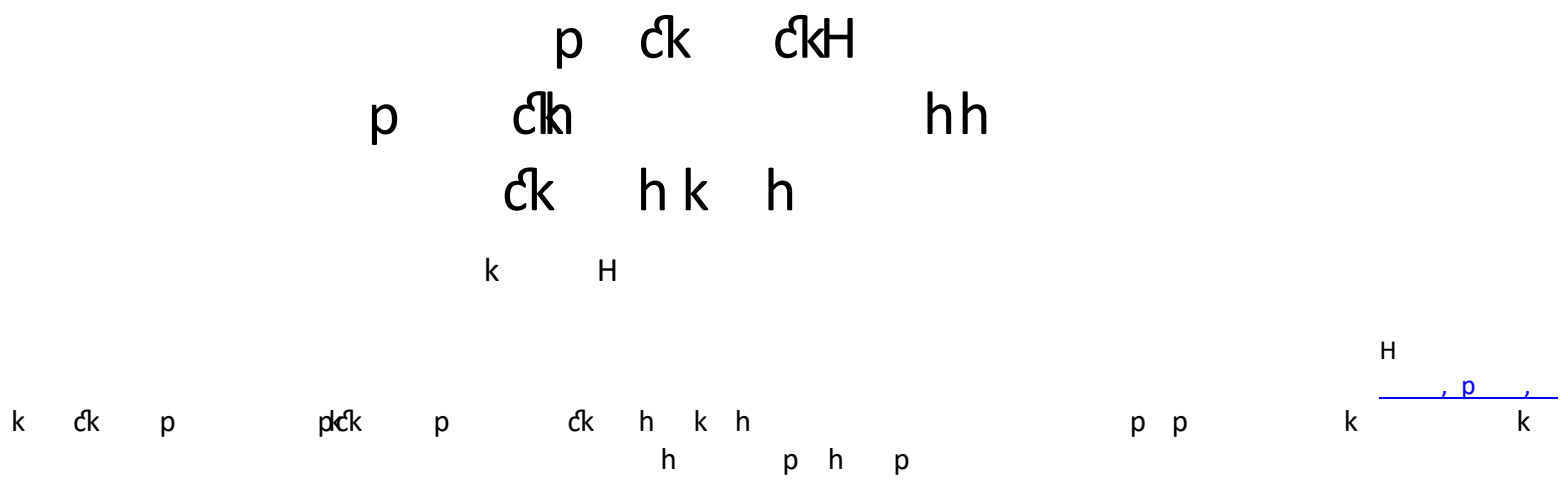

Comparing US and Spanish student teachers' perceptions on social media

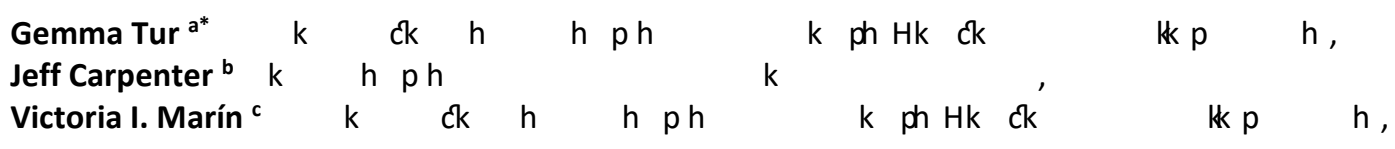


$\mathrm{p}, \mathrm{p} \mathrm{p}, \mathrm{p} \mathrm{H}, \mathrm{ph} \quad \mathrm{ck} \mathrm{h}$ ck

Trends and Issues Proceedings on Humanities and Social Sciences. It (,

p. p h hk clk, New

1. Introduction

hk clk h tck $p$ ck ck $p h \quad k p h$

$k h \quad h \quad c k$

ck h, pclk

, $p h k p$

h ck ck ck k $\mathrm{H}^{\prime}$ p $\mathrm{h}$

$p c k \quad h \quad p \quad p$,

ck h k h

$k \quad h \quad, \quad c k \quad p c l k$

$\begin{array}{llllllllllllllll}k & k H & p & P & p & H & p & h & & p\end{array}$

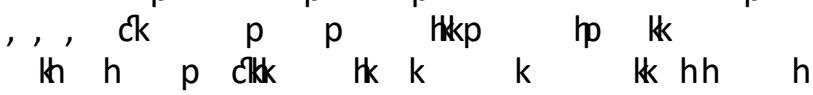

$h \mathrm{H} \quad \mathrm{p} \quad \mathrm{p}$ hth $\mathrm{ck} h \mathrm{~h}$ ch $\mathrm{p}$

p c

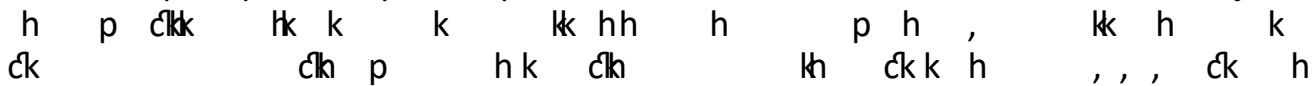

$P \quad p \quad p h k$ Clk ck

\& $\mathrm{p}$

Hk H $\quad p \quad c k h$ $\mathrm{kh}$

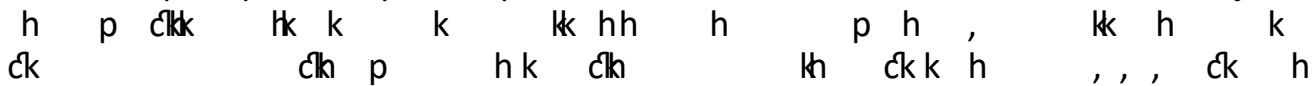

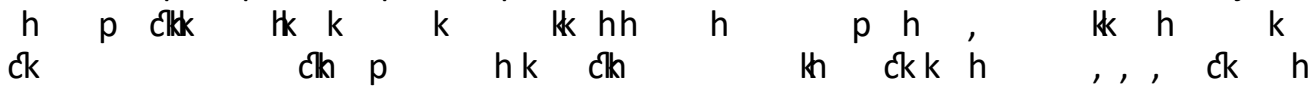

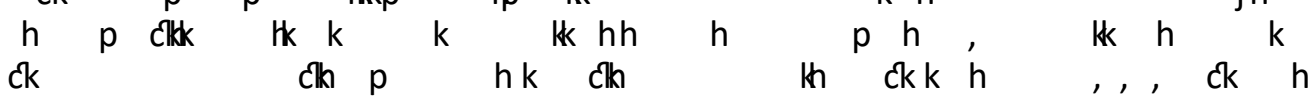

$p h \quad h$

Table 1. Use of various social media

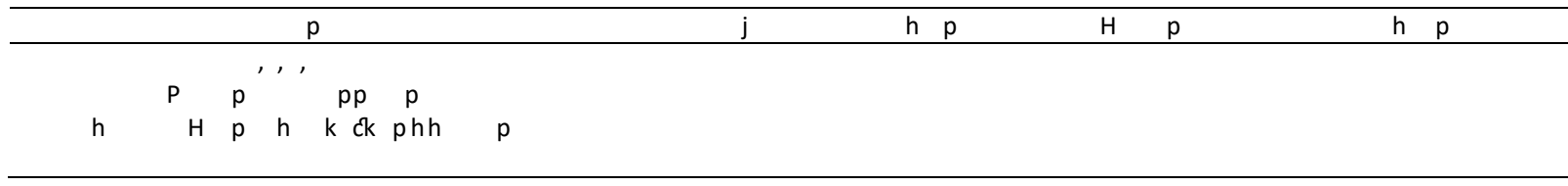

$\mathrm{h} \quad \mathrm{ck} \quad \mathrm{kp} \quad \mathrm{hk}$ clh $\quad \mathrm{ck}$

p $p h \quad k \quad p c k \quad h \quad k h, H h$

$p h \quad p \quad h \quad h \quad h \quad p h h \quad p \quad j c k$

, $h$ pkp $p$ ckp ck ck $p \quad p$

ck ck $h$ phk $k$ p $p \quad p \quad p \quad k \quad p j$

$\begin{array}{lllllllll}p & p & & & c k & h & c k \\ h & c k & h & p & h & k & c k & p\end{array}$

$h p p p p$ p

tckp $h \quad p \quad h$

hh $k h$ h $p$,

\section{Social media in Education}

ck h hk clk $\mathrm{h}$

$h$ hh ck ph $\quad h k$ clk $p$ ckh $p$

$p h \quad p h$ ck $p \quad p \quad k p$ Clk $p$

ph $p$ p clh $p k h$ h

lk ck kph pph ? ck phh h

$h k$ ck $k$ pkh k $h \quad$, ckh

h k clh h k clk

$\mathrm{k} p \quad \mathrm{k}$ ck $\mathrm{ph}$

$\mathrm{h}$

h $\mathrm{k}$
Ih hhh H p p j ck $p p$ pclk k ph

$\begin{array}{llllll}h & h & c k & k & p h & \\ h k & C l k h & h & \end{array}$

$\begin{array}{llllll}h & h & c k & k & p h\end{array}$, ph th ck ck $p h k p d k \quad k$ phh $k h j h$ jhk pp p. $p h$ hck k $p$ p

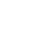

\subsection{Social media in the educational systems of the U.S.A. and Spain}

$h k$ clk ph ck h h , , ck h h clk $p \quad p, H \quad h$ ck

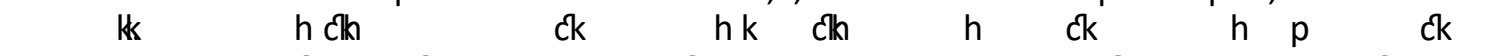
$p p k h \quad h \quad c k$ ckk $p h \quad c k \quad k \quad$, hk ck $p \quad p h p p \quad p \quad$ ? $h p h \quad h \quad$ h $p$ ck $h$ k $h k$ Clk $k$ th $p h h \quad h \quad h \quad k$ hh $p h \quad h \quad h \quad c k$ k $p h \quad p \quad p \quad p$

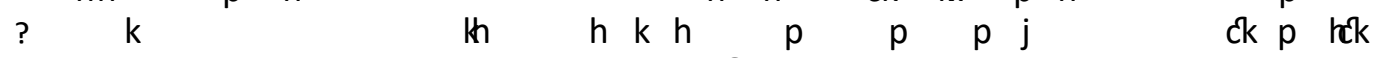

$p \quad p \quad p h \quad h k$ ck $k \quad h \quad p h \quad k j$ $p \quad c k h k$ dk $h$, $h \quad h \quad h \quad h \quad p \quad h{ }^{\prime}$

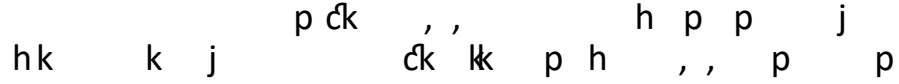

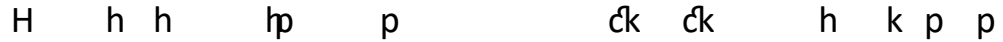

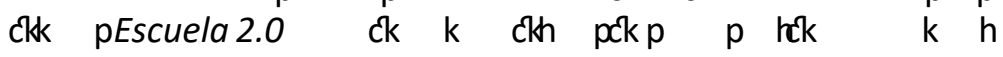

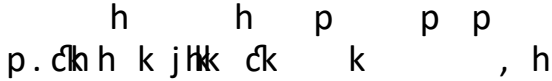
ph $p h$ ck 
$\mathrm{p}, \mathrm{p} \mathrm{p}, \quad \mathrm{ph} \mathrm{H}, \quad \mathrm{ph} \quad \mathrm{ck} h \mathrm{~h}$

Trends and Issues Proceedings on Humanities and Social Sciences. It I,

p. p h hk clk, New

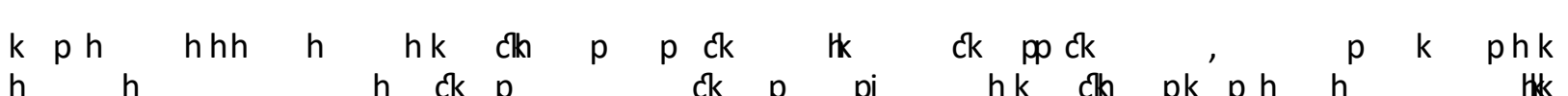

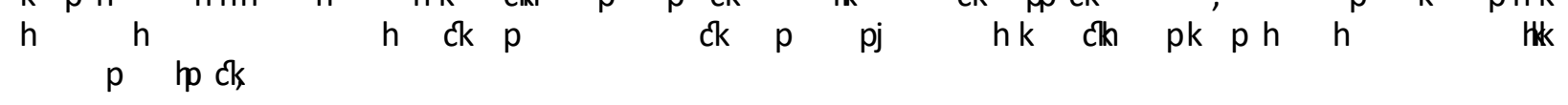

\section{Research}

\subsection{Context and Participants}

ck pph ck h

$\mathrm{Hk}$ Ck h h ckh k h ph h

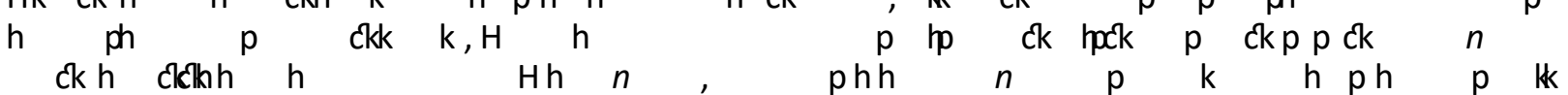

I $p$ ckHh $h p h$

k ph $\begin{array}{cccccccc}c k p k & h & p & p & c k & p & p & p\end{array}$

3.2. Learning activity

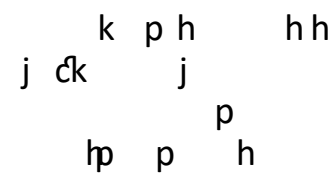
$\begin{array}{cccccccc}\text { pph ck } & p & & h p & k & h & k & p \\ j k & k k & k & k & & & c k & p h\end{array}$
h p, ck $p$

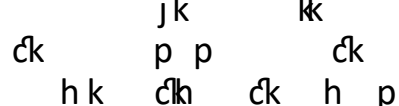

\subsection{Research questions}

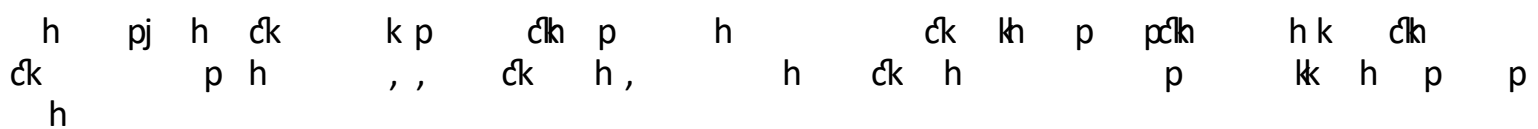

$$
\begin{aligned}
& \text {, } p \quad p \text { clh } p \quad h \quad \text {, } c k \text { h ck } \quad \text { hk clk! }
\end{aligned}
$$

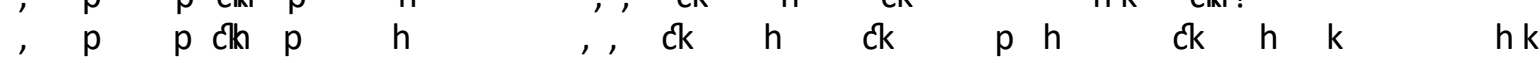

\subsection{Methodology and Instrument}

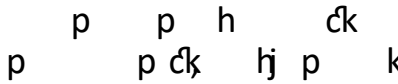

$$
p h \quad h \quad p
$$
$\begin{array}{lllll}\mathrm{p} & \mathrm{h} & \mathrm{p} & \mathrm{h} & \mathrm{h}\end{array}$
h h ck th $h$ ck

$\begin{array}{cccc}p h \quad p h & h & p \\ c k & k p h c k & p \\ c k & p & p c k\end{array}$
Hk ck pho
$p \quad c k p j$ ph ck
$h \mathrm{~h}$ ith $p$ th $k$ k $\stackrel{p}{p} \quad c k h^{\prime}$

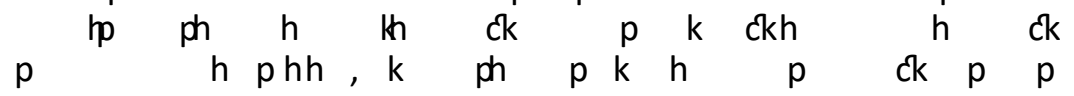 ck $\quad p k$ h $p$ pck

\section{Results}

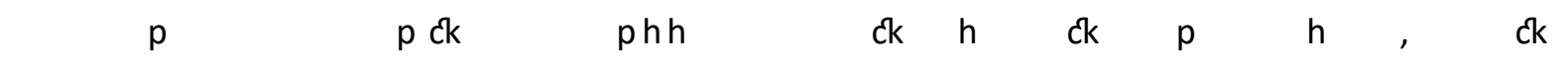

4.1. Research Question 1: Are there differences in the way U.S. and Spanish pre-service teachers use Social Media?

Table 2. What social media services do you use?

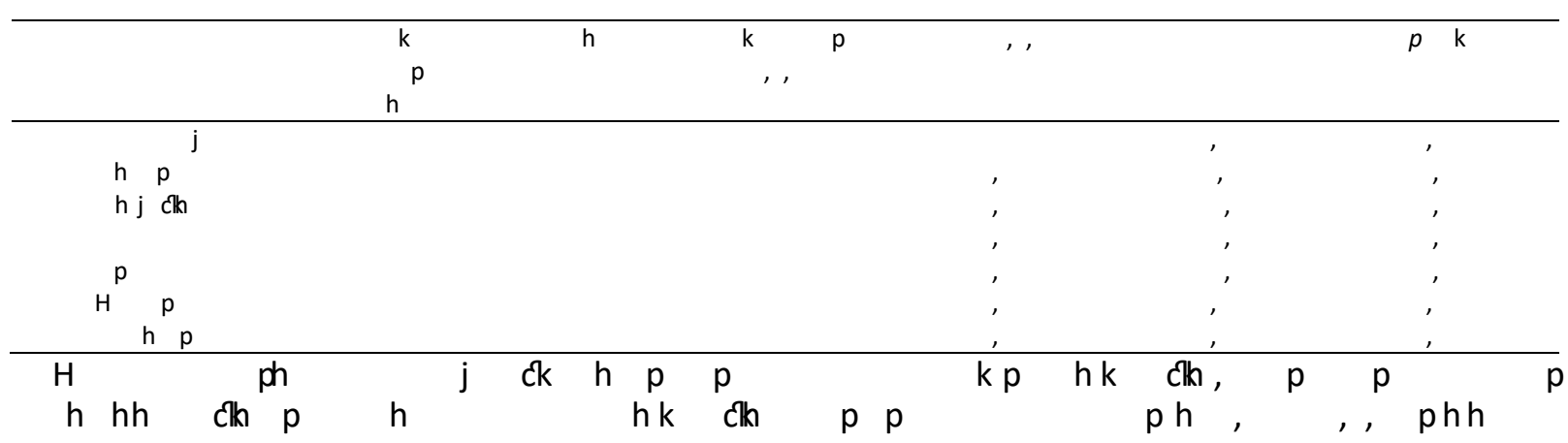


$\mathrm{p}, \mathrm{p} \mathrm{p}, \quad \mathrm{ph} \mathrm{H}, \quad \mathrm{ph} \quad \mathrm{ck} h \mathrm{~h}$

Trends and Issues Proceedings on Humanities and Social Sciences. It I,

p. p h hk Clk, New
$\begin{array}{llllll}p & k & h & h & h & p \\ & c k & & j,\end{array}$
$h$ j clk $\underset{k}{ }$ ck $p$
$p c k$
He h phh
ph $h \mathrm{~h}$
$p_{p} \quad p H i k$ ck.
pk hk clk,

4.2. Research Question 2: Are there differences in the way U.S. and Spanish pre-service teachers perceive the educational use of Twitter and other social media?

Table 3. Which of the following social media services do you think have educational uses? (Check all that apply)

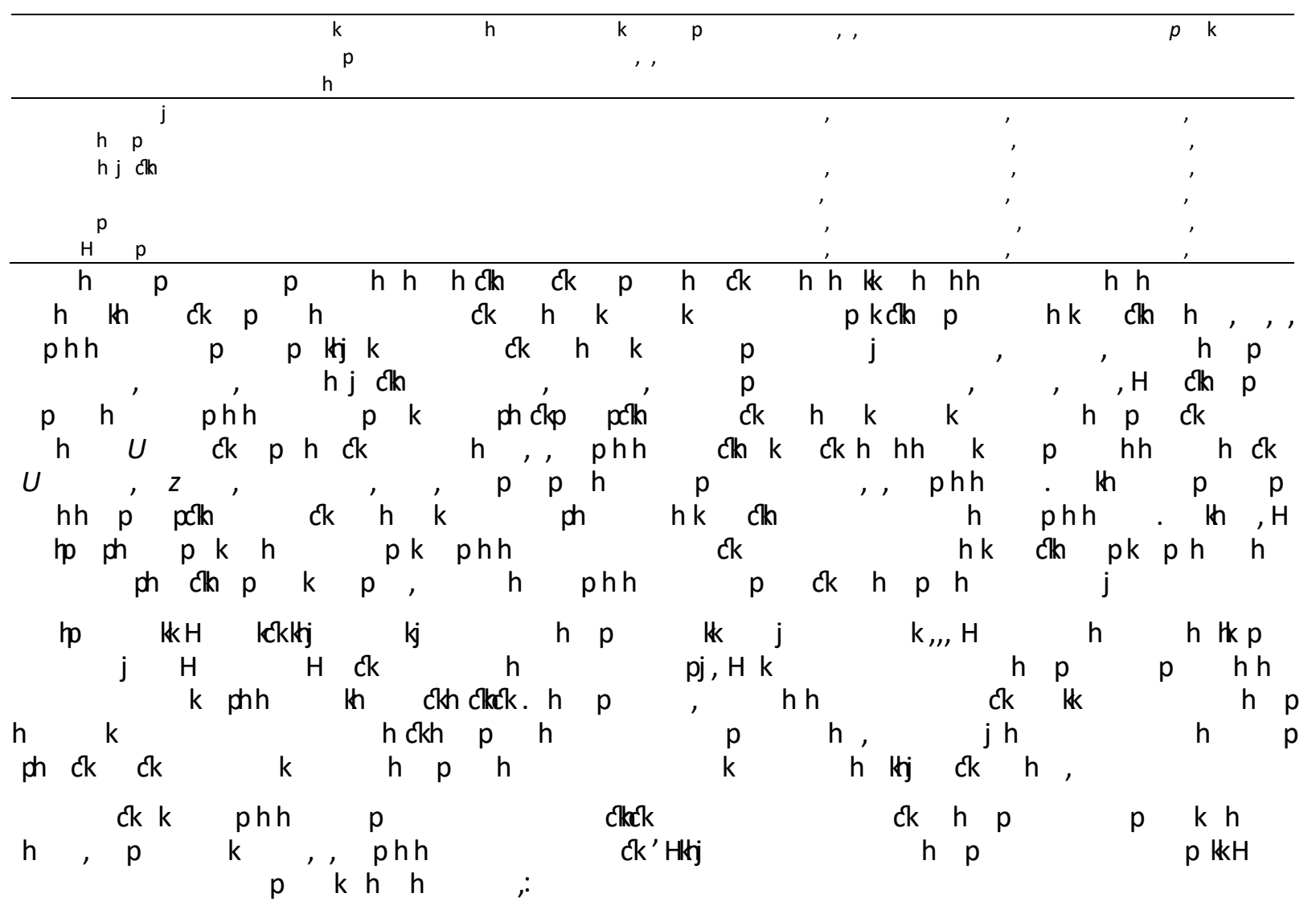

\section{Discussion and Conclusion}

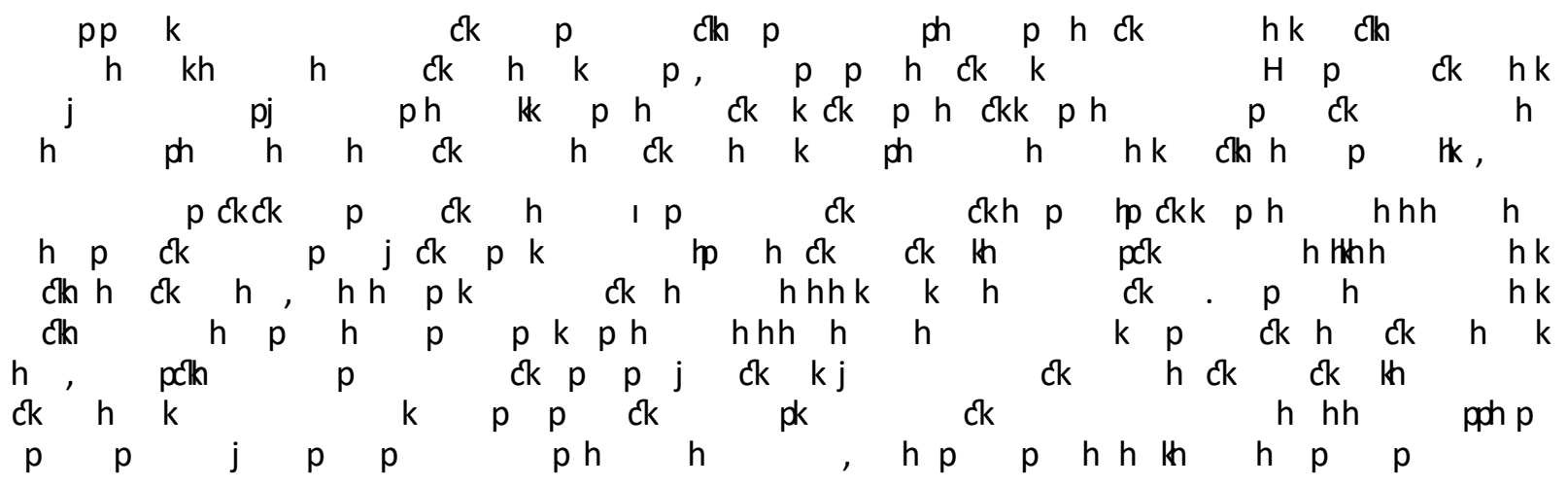


$\mathrm{p}, \mathrm{p} \quad \mathrm{p}, \mathrm{ph} \mathrm{H}, \mathrm{ph} \quad \mathrm{ck} \mathrm{h}$ ck

Trends and Issues Proceedings on Humanities and Social Sciences. It (, p. $p h$ hk Clk, New

, $\mathrm{k} k \mathrm{k}$

? $\mathrm{lp}$ Ck $\mathrm{p}$

ck ck

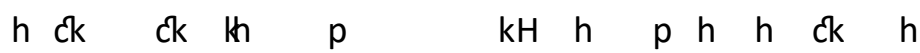

phlk ih $h \quad p \quad p d k$ Clk $p \quad p \quad p$

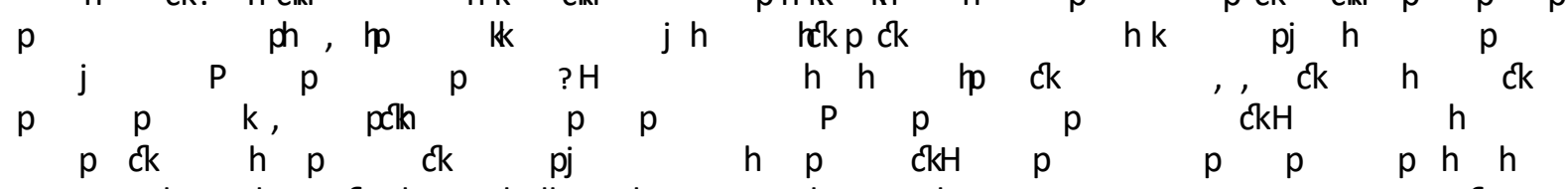

, $h \quad h \quad c k h \quad h k h \quad h \quad p \quad k \quad h \quad p \quad p$

h $p$ ck pj , k

$p \quad p \quad p h \quad k \quad p$

phh h h h clk ck

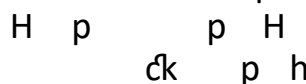

$p k \quad p \quad h \quad p$

h $h$ ih

h $p$

h h $\quad$ Clk

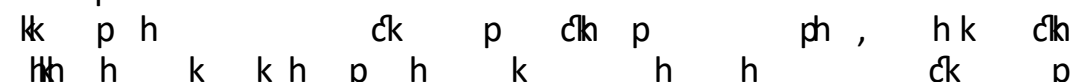

kck $k p$

h thh h ck ck k phh ckh h h p hhh $h \quad h \quad p h, H \quad k k$ k

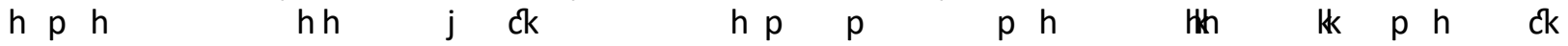

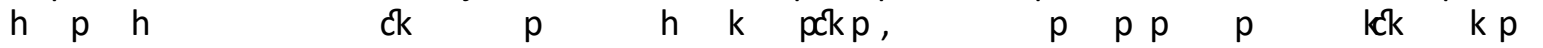

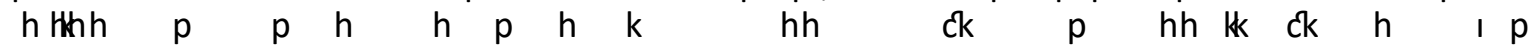
k $p h$ ck ph $p k p h \quad h$ h k pk hk clk ph k k phh ck k tek $h \quad$ pdck hh $p$ ck $p, \quad$ ck k k k

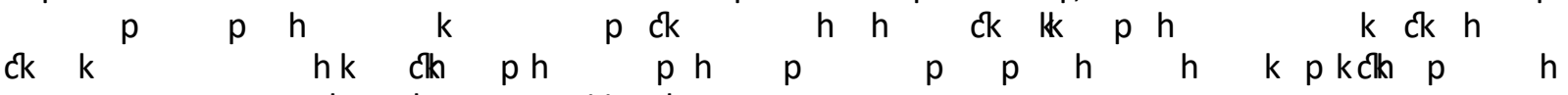
$p \quad P \quad h \quad k \quad j j p h$

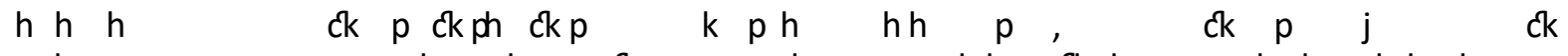

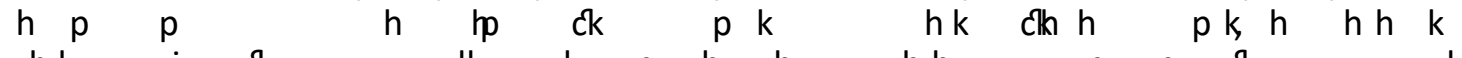

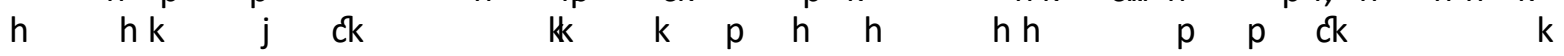
$p k \quad p k p \quad c k \quad p h k p \quad p h \quad, \quad p \quad p \quad p \quad p h$ Clk Hk kp

$p \quad p$,

\section{References}

$\mathrm{p}, \mathrm{k} \mathrm{p} \quad \mathrm{ck} \mathrm{h} \quad \mathrm{p} \mathrm{h} \quad \mathrm{ck} \mathrm{k} \mathrm{H} \quad \mathrm{k} \quad \mathrm{p} \quad \mathrm{kp} \quad$ Clh Ck , Revista

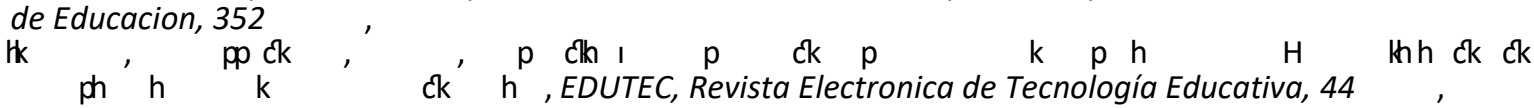
$\mathrm{p} p, \quad \mathrm{p}, \mathrm{h} p \mathrm{~h} \quad \mathrm{p} \quad \mathrm{k} \mathrm{h} \mathrm{k} \mathrm{h}$, International Journal of Social Media and Interactive Learning Environments, 2

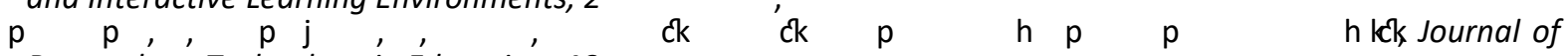
Research on Technology in Education, $46 \quad \mathrm{p} \quad \mathrm{hp} k \mathrm{~h}$ ck $\mathrm{p} p \mathrm{p}$ hck k

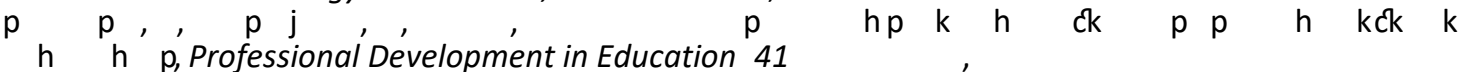

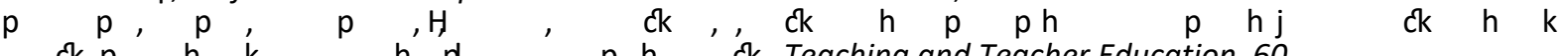

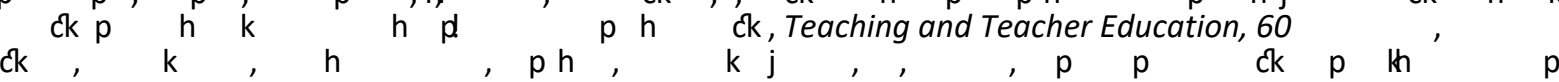
$\mathrm{p}$ ' $\mathrm{h}$ kck 'k , Journal of Teacher Education, 60 '

$\mathrm{p}, \mathrm{h} p \mathrm{pk}, \mathrm{P}, \mathrm{pH}, \mathrm{hp} k \mathrm{~h}$ h $\mathrm{p}$ ck $\mathrm{h}$

Hh $h p^{\prime} k$ ck $p$ ph cḱ $p h$ ! Computers \& Education, 55

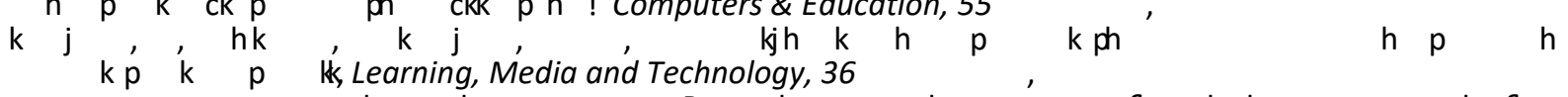

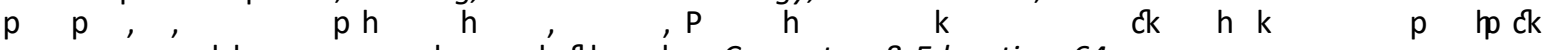

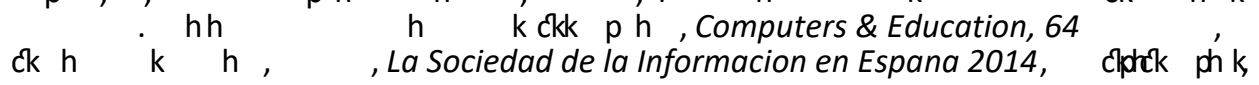


$\mathrm{p}, \mathrm{p} \mathrm{p}, \mathrm{p} \mathrm{H}, \quad \mathrm{ph} \quad \mathrm{ck} h \mathrm{~h}$

Trends and Issues Proceedings on Humanities and Social Sciences. It (,

p. p h

h k clk, New

Trends and Issues Proceedings on Humanities and Social Sciences. Ih 1
$\mathrm{p}_{\text {ith }} \mathrm{P}$ ck $\mathrm{k}_{\mathrm{p}}^{\prime} \quad \mathrm{p}$ j ,
pp International, ' 51
$\mathrm{k} \mathrm{j}, \stackrel{\mathrm{h}}{\mathrm{p}, \text { Computers \& Education, } 51}$
ph $p \quad k$
p. ck h k pph $\mathrm{k}$
$\mathrm{p} \mathrm{h}$
phh k h'jh, Educational Media

$$
\text { h p p p k ph ck }
$$
, The English $\mathrm{p},{ }^{\prime} \mathrm{p}, \mathrm{p}$

$\mathrm{H}$

$\mathrm{p} \mathrm{h} \mathrm{k} \mathrm{Ck} \mathrm{phh} \mathrm{p} \quad \mathrm{H} \quad$ VI Estudio Redes Sociales de IAB Spain, $\mathrm{P} p \mathrm{ph} \quad \mathrm{pk}$

$\mathrm{j}$
Journal of Computer Assisted Learning, 27 h of Computer-Mediated Communication 13

h $\mathrm{P}, \mathrm{k}$ h

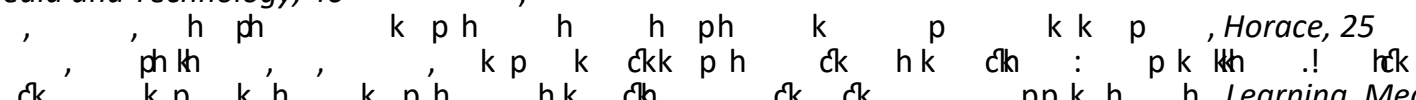

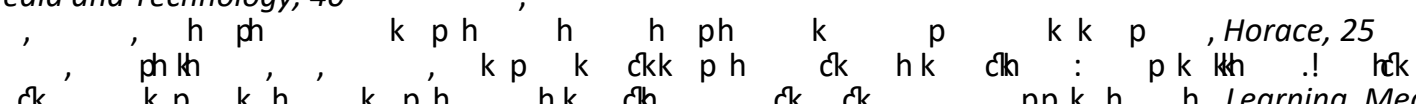

$\mathrm{p} p \mathrm{p}$ ih $\mathrm{h} h$

p $\quad \mathrm{P}$ k $\mathrm{k} \quad \mathrm{kClk} k \mathrm{~h} \quad \mathrm{~h}$

$\mathrm{p} \quad \mathrm{hk}$

h k pj h, Journal

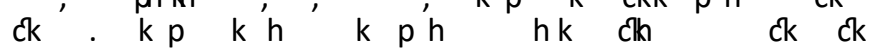
Technology, 41
ik, , , $\mathrm{h}$ cीkp $\mathrm{h}$ $\mathrm{k} h$ j Clk h k $p p k \mathrm{~h}$

hk pjh $\mathrm{h}$, Learning, th $\mathrm{p} h$ h $\mathrm{k}$ k $\mathrm{p}$,Educational Media International 51

$\mathrm{k},, \mathrm{kh} \mathrm{ck} \mathrm{h} \mathrm{H} \mathrm{h}$ ck ph $\mathrm{k}$ ck $\mathrm{ck} \mathrm{k}^{\prime}$, Campus Virtuales, 1

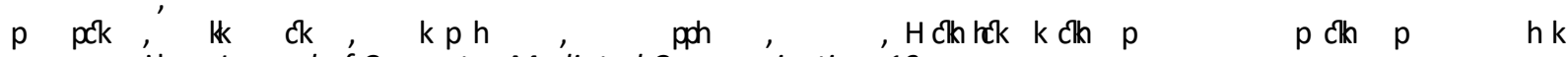
pj h , Journal of Computer-Mediated Communication, 19

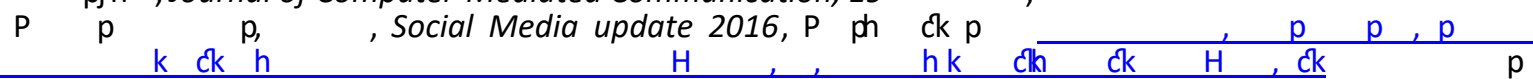

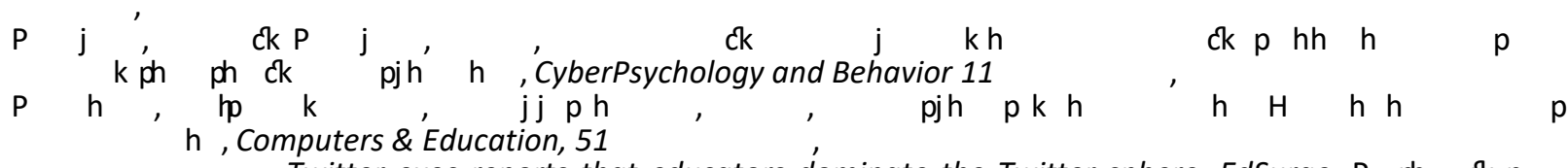
, Twitter exec reports that educators dominate the Twitter-sphere. EdSurge, P ph ck p

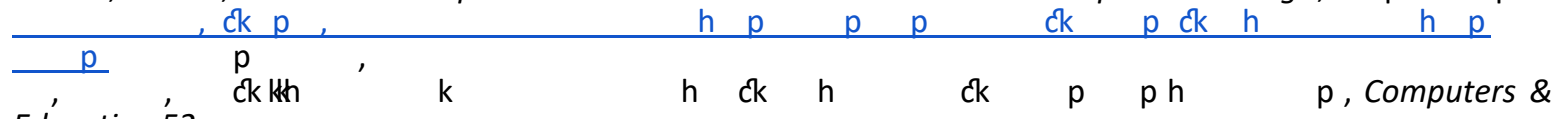

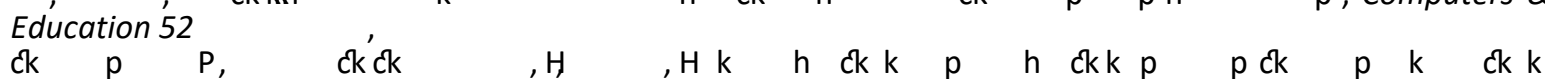

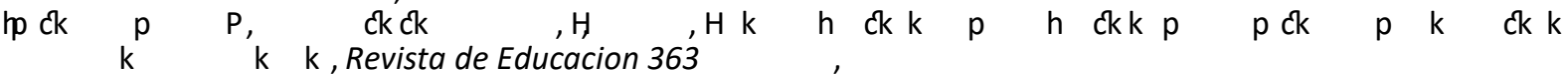

$\mathrm{h} \mathrm{ph} \quad \mathrm{h}, \quad, K-12$ teachers uncertain about how to connect with students and parents via social media, $\mathrm{P}$ ph Ck $\mathrm{p}, \mathrm{p}, \mathrm{ck} \quad \mathrm{pk} \quad \mathrm{p}$

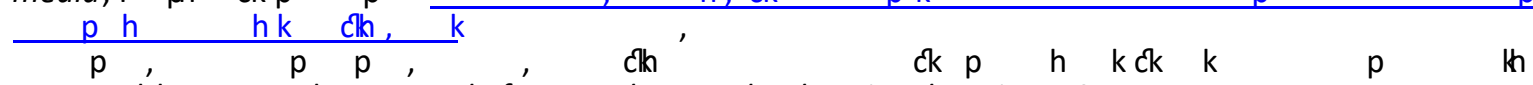
hh $\mathrm{p} h$, Journal of Research on Technology in Education, 40

$\mathrm{k}, \mathrm{H} \quad \mathrm{h} \mathrm{h} \quad \mathrm{h} \quad \mathrm{p} h \mathrm{~h}$ pkk $\mathrm{ck} \quad \mathrm{p} \quad \mathrm{h}$, Journal of Teacher Education, 64 